\title{
Clustering Cycles into Cycles of Clusters ${ }^{\star}$
}

\section{Extended Abstract}

\author{
Pier Francesco Cortese, Giuseppe Di Battista, \\ Maurizio Patrignani, and Maurizio Pizzonia \\ Dipartimento di Informatica e Automazione - Università Roma Tre, Italy \\ \{cortese,gdb,patrigna,pizzonia\}@dia.uniroma3.it
}

\begin{abstract}
In this paper we study the clustered graphs whose underlying graph is a cycle. This is a simple family of clustered graphs that are "highly non connected". We start by studying 3-cluster cycles, that are clustered graphs such that the underlying graph is a simple cycle and there are three clusters all at the same level. We show that in this case testing the c-planarity can be done efficiently and give an efficient drawing algorithm. Also, we characterize 3-cluster cycles in terms of formal grammars. Finally, we generalize the results on 3-cluster cycles considering clustered graphs that at each level of the inclusion tree have a cycle structure. Even in this case we show efficient c-planarity testing and drawing algorithms.
\end{abstract}

\section{Introduction}

Consider the following problem. A cycle is given where each vertex has a label. Is it possible to add new edges so that: (i) the new graph (i.e. cycle plus new edges) is planar and (ii) for each label, the subgraph induced by the vertices with that label is connected? An example is in Fig. 1.a. In this case the problem admits a solution, depicted in Fig. 1.b.

In this paper we tackle problems of the above type. Such kind of problems arise in the field of clustered planarity [9,8]. Given a graph, a cluster is a non empty subset of its vertices. A clustered graph consists of a graph $G$ and a rooted tree $T$ such that the leaves of $T$ are the vertices of $G$. Each node $\nu$ of $T$ corresponds to the cluster $V(\nu)$ of $G$ whose vertices are the leaves of the subtree rooted at $\nu$. The subgraph of $G$ induced by $V(\nu)$ is denoted as $G(\nu)$. An edge $e$ between a vertex of $V(\nu)$ and a vertex of $V-V(\nu)$ is said to be incident on $\nu$. Graph $G$ and tree $T$ are called underlying graph and inclusion tree, respectively. A clustered graph is connected if for each node $\nu$ of $T$ we have that $G(\nu)$ is connected.

In a drawing of a clustered graph each vertex of $G$ is a point and each edge is a simple curve between its end-vertices. For each node $\nu$ of $T, G(\nu)$ is drawn inside a

\footnotetext{
* Work partially supported by European Commission - Fet Open project COSIN - COevolution and Self-organisation In dynamical Networks - IST-2001-33555, by European Commission Fet Open project DELIS - Dynamically Evolving Large Scale Information Systems - Contract no 001907, by "ALGO-NEXT: Algorithms for the Next Gen. Internet and Web", MIUR Programmi di Ricerca Scientifica di Rilevante Interesse Nazionale, and by "The Multichannel Adaptive Information Systems (MAIS) Project", MIUR-FIRB.
} 


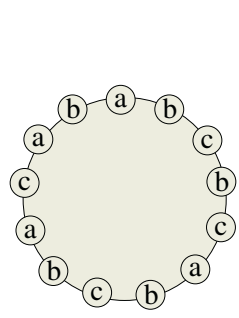

(a)

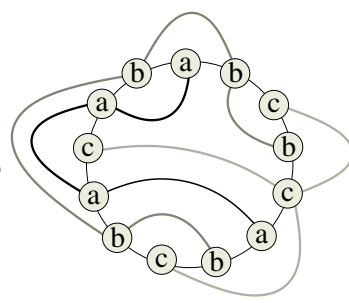

(b)

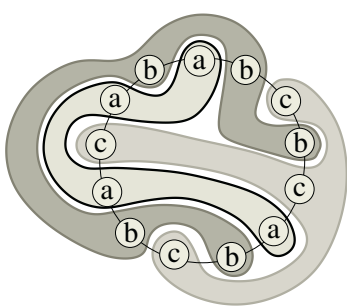

(c)

Fig. 1. (a) An example of a cycle with labels in $\{a, b, c\}$. (b) The cycle with extra edges. (c) The corresponding clustered drawing of the cycle.

simple closed region $R(\nu)$ such that: (i) for each node $\mu$ of $T$ that is neither an ancestor nor a descendant of $\nu, R(\mu)$ is completely contained in the exterior of $R(\nu)$; (ii) an edge $e$ incident on $\nu$ crosses the boundary of $R(\nu)$ exactly once. We say that edge $e$ and region $R$ have an edge-region crossing if both endpoints of $e$ are outside $R$ and $e$ crosses the boundary of $R$. A drawing of a clustered graph is c-planar if it does not have edge crossings and edge-region crossings. A clustered graph is $c$-planar if it has a c-planar drawing.

Consider again the example of Fig. 1 according to the above definitions. The cycle is the underlying graph of a clustered graph. Vertices with the same label are in the same cluster. The inclusion tree consists of a root with three children, denoted $a, b$, and $c$. The children of node $x$ are the vertices labeled $x$. The edges added to the cycle are used to "simulate" the closed regions containing the clusters (See Fig. 1.c). In this paper we call saturator such set of edges. The clustered graph of the example is cplanar. Further, the problem of adding extra edges to a labeled cycle admits a solution iff the corresponding clustered graph is c-planar. Observe that the clustered graph of the example is not connected.

Clustered planarity, because of its practical impact and because of its theoretical appeal, attracted many research contributions. Feng, Cohen, and Eades devised the first polynomial time c-planarity testing algorithm for connected clustered graphs [9]. A planarization algorithm for connected clustered graph is shown in [5]. However, the complexity of the problem for a non connected clustered graph is still unknown.

A contribution on this topic has been given by Gutwenger et al. that presented a polynomial time algorithm for c-planarity testing for almost connected clustered graphs [10]. In almost connected clustered graphs either all nodes corresponding to non connected clusters are in the same path in $T$ starting at the root of $T$, or for each non connected cluster its parent and all its siblings are connected. Also, the works in [1,2] by Biedl, Kaufmann, and Mutzel can be interpreted as a linear time c-planarity test for non connected clustered graphs with exactly two clusters at the same level.

Another contribution studying the interplay between c-planarity and connectivity has been presented in [3] by Cornelsen and Wagner. They show that a completely connected clustered graph is c-planar iff its underlying graph is planar. A completely connected clustered graph is so that not only each cluster is connected but also its complement is connected. 
In this paper we study the clustered graphs whose underlying graph is a cycle. This is a simple family of clustered graphs that are "highly non connected". The paper is organized as follows.

Section 2 contains preliminaries. In Section 3 we study 3-cluster cycles, that are clustered graphs such that the underlying graph is a simple cycle and there are three clusters all at the same level. We show that, in this case, testing the c-planarity can be done efficiently. We also give an efficient drawing algorithm. Further, we show that in this specific case if the c-planarity problem admits a solution then a saturator exists that is composed only by simple paths. In Section 4 we generalize the results on 3cluster cycles considering clustered graphs that on each level of the inclusion tree have a cycle structure. Even in this case we show efficient c-planarity testing and drawing algorithms. Section 5 contains conclusions and open problems.

\section{Preliminaries}

We assume familiarity with connectivity and planarity of graphs $[7,6]$. We also assume familiarity with formal grammars [11].

Given a c-planar non connected clustered graph $C(G, T)$, a saturator of $C$ is a set of edges that can be added to the underlying graph $G$ so that $C$ becomes connected without loosing its c-planarity. Finding a saturator of a clustered graph is important since it allows to apply to $C$ the same drawing techniques that have been devised for connected clustered graphs.

We call 3-cluster cycle a clustered graph such that the underlying graph is a simple cycle and there are exactly three clusters all at the same level (plus the root cluster). In a 3-cluster cycle the inclusion tree consists of a root node with three children and each vertex of the underlying cycle is a child of one of these three nodes. Given a 3-cluster cycle, we associate a label in $\{a, b, c\}$ to each of the three clusters.

Consider a 3-cluster cycle and arbitrarily select a starting vertex and a direction. We can visit the cycle and denote it by the sequence $\sigma$ of labels associated with the clusters encountered during the visit. The same clustered cycle is also denoted by any cyclic permutation of $\sigma$ and by any reverse sequence of such permutations. We use Greek letters to denote general sequences and Roman letters to identify single-character sequences. Given a sequence $\sigma$, we denote with $\bar{\sigma}$ its reverse sequence.

A non c-planar c-cluster cycle is $a b c a b c$, while a c-planar one is abcbac.

It is easy to see that repeated consecutive labels can be collapsed into a single label without affecting the c-planarity property of a 3-cluster cycle. Hence, in the following we consider only 3 -cluster cycles where consecutive vertices belong to distinct clusters. Also, since clusters can not be empty, in a 3-cluster cycle at least one occurrence of each label can be found.

We assign a cyclic order to the clusters so that $a \prec b, b \prec c$, and $c \prec a$. A sequence $\sigma$ is monotonic increasing (decreasing) if for each pair $x, y$ of consecutive labels of $\sigma$ $x \prec y(y \prec x)$. A sequence is cyclically increasing (decreasing) monotonic if all its cyclic permutations are increasing (decreasing) monotonic.

Given a 3-cluster cycle $\sigma$, Balance $(\sigma)$ is a number defined as follows. Select a starting vertex and a direction. Set counter $c$ to zero. Visit $\sigma$ adding (subtracting) one 
unit to $c$ when passing from $x$ to $y$, where $x \prec y(y \prec x)$. Observe that, when the starting vertex is reached again, $c$ is a multiple of 3 that can be positive, negative, or zero. If we selected a different starting vertex, while preserving the direction, we would obtain the same value. On the contrary, if $\sigma$ was visited in the opposite direction the opposite value would be obtained for $c$. Balance $(\sigma)=|c|$. For example, Balance $(a b a b c)=3$ and Balance $($ cbacba $)=6$.

Observe that, when representing a 3-cluster cycle with a sequence of labels, by reading the sequence from left to right, we implicitly choose a direction for visiting the cycle. For simplicity, we adopt the convention of representing a 3-cluster cycle with a sequence $\sigma$ such that, when the vertices of the cycle are visited according to the order induced by $\sigma$, a non negative value for $c$ is obtained.

\section{Cycles with Three Clusters}

In this section we address the problem of testing the c-planarity of a 3-cluster cycle. The following lemma introduces transformations that can be used to simplify 3-cluster cycles without affecting their c-planarity properties.

Lemma 1. Let $\sigma=\sigma_{1} x \alpha y \bar{\alpha} x \alpha y \sigma_{2}$ be a 3-cluster cycle such that $\sigma_{1}, \sigma_{2}$, and $\alpha$ are possibly empty and $x \alpha y$ is monotonic. The 3-cluster cycle $\sigma^{\prime}=\sigma_{1} x \alpha y \sigma_{2}$ is c-planar if and only if $\sigma$ is c-planar. Balance $(\sigma)=\operatorname{Balance}\left(\sigma^{\prime}\right)$.

Proof Sketch: Suppose there exists a c-planar drawing of $\sigma^{\prime}$. The black line in Fig. 2.a shows an example of such a drawing for the portion concerning subsequence $x \alpha y$. Such a drawing can be modified by replacing the edge between $y$ and the first vertex of $\sigma_{2}$ with the sequence $\bar{\alpha} x \alpha y$. Such sequence can be drawn arbitrarily close to $x \alpha y$ preserving c-planarity. Finally, the just added instance of $y$ may be connected to the first vertex of $\sigma_{2}$. The result is shown in Fig. 2.a where the added part is drawn gray.

Now, suppose that there exists a c-planar drawing of $\sigma$. Fig. 2.b shows an example of such a drawing for the part concerning subsequence $x \alpha y \bar{\alpha} x \alpha y$. The inlet formed by $x \alpha y \bar{\alpha} x$ may contain parts of $\sigma$ that are denoted by $Q$ in Fig. 2.b. The parts of $\sigma$ that are contained in the inlet formed by $y \bar{\alpha} x \alpha y$ are denoted by $P$. The embedding of $P$ and $Q$ may be rearranged preserving c-planarity as in Fig. 2.c. Path $\bar{\alpha} x \alpha y$ can now

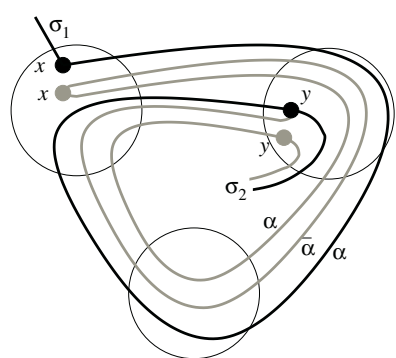

(a)

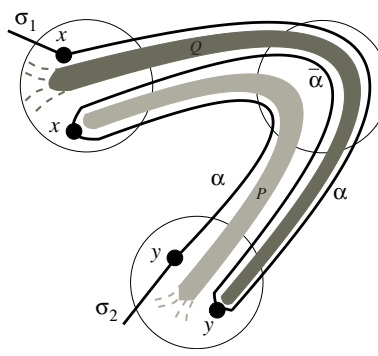

(b)

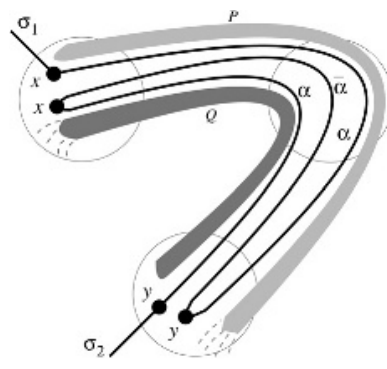

(c)

Fig. 2. Illustration of the proof of Lemma 1. (a) Necessary condition. (b) and (c) Sufficient condition. 
be deleted and substituted by an edge connecting vertex $y$ with the first vertex of $\sigma_{2}$. Finally, observe that, since we have removed from $\sigma$ two monotonic sub-sequences, one increasing and one decreasing, with the same length, $\operatorname{Balance}\left(\sigma^{\prime}\right)=\operatorname{Balance}(\sigma)$.

For example, Lemma 1 allows to study the c-planarity of cabcab instead of the cplanarity of cabcacbabcab (by taking $\sigma_{1}=c, x=a, \alpha=b c, y=a$, and $\sigma_{2}=b$ ).

Lemma 2. Let $\sigma$ be a 3-cluster cycle. There exists a 3-cluster cycle $\sigma^{\prime}$ such that: Balance $\left(\sigma^{\prime}\right)=$ Balance $(\sigma), \sigma^{\prime}$ is c-planar iff $\sigma$ is c-planar, and either $\sigma^{\prime}$ is cyclically monotonic or $\sigma^{\prime}=x \alpha y \beta$, where

1. $\alpha$ and $\beta$ are non empty,

2. $x \alpha y$ is maximal monotonic increasing, and

3. y $\beta x$ is maximal monotonic decreasing.

Because of space limitation, the proof for this Lemma is omitted. This proof is based on the main idea of repeatedly applying Lemma 1 starting from the shortest monotonic subsequences [4].

The following two lemmas (Lemma 3 and Lemma 4) study the c-planarity of the simple families of 3-cluster cycles cited in Lemma 2.

Lemma 3. A 3-cluster cycle $\sigma$ such that $\sigma$ is cyclically monotonic is c-planar if and only if Balance $(\sigma)=3$.

Proof Sketch: Since $\sigma$ is monotonic we have that $\operatorname{Balance}(\sigma) \neq 0$. Recall that $\operatorname{Balance}(\sigma)$ is a multiple of 3 . If $\operatorname{Balance}(\sigma)=3$, then it can only be the case that $\sigma=a b c$ or $\sigma=b c a$ or $\sigma=c a b$ and it is trivial to see that $\sigma$ is c-planar.

Suppose that $\operatorname{Balance}(\sigma) \geq 6$. We show that $\sigma$ is not c-planar. Suppose by contradiction that there exists a c-planar drawing $\Gamma_{\sigma}$ of $\sigma$. Consider the vertices $v_{1}, v_{2}, v_{3}$, $v_{4}, v_{5}$ and $v_{6}$ of $\sigma$ as drawn in $\Gamma_{\sigma}$ (see Fig. 3.b). The two edges incident to $v_{4}$ separate $v_{1}$ from the rest of the vertices of its cluster. Thus, it is possible to add an edge $\left(v_{1}, v_{4}\right)$ preserving the planarity of the drawing. For similar reasons, it is possible to add the edges $\left(v_{2}, v_{5}\right)$ and $\left(v_{3}, v_{6}\right)$. A contradiction arises from the fact that a subdivision of a $K_{3,3}$ can be found in the drawing. Consider, the vertices $v_{1}, v_{2}, v_{3}, v_{4}, v_{5}$ and $v_{6}$. Vertex $v_{1}$ is connected to $v_{6}$ with a path in $\sigma$ and it is directly connected to $v_{2}$ and $v_{4}$. Vertices $v_{3}$ and $v_{5}$ are directly connected to $v_{2}, v_{4}$, and $v_{6}$.

Lemma 4. Let $\sigma=x \alpha y \beta$ be a 3-cluster cycle, where $\alpha$ and $\beta$ are non empty, x $\alpha y$ is maximal monotonic increasing, and $y \beta x$ is maximal monotonic decreasing. We have that $\sigma$ is c-planar iff Balance $(\sigma)$ is in $\{0,+3\}$.

Proof Sketch: Let Balance $(\sigma)=3 k$, with $k$ non negative integer. Suppose $k$ is equal to 0 or 1 . A c-planar drawing of $\sigma$ can be constructed by placing the vertices on three half-lines as in the examples shown in Fig. 4.a and 4.b, respectively. The vertices of each half-line can be enclosed into a region representing their cluster.

Suppose that $k>1$. We show that $\sigma$ is not c-planar. Suppose for a contradiction that $\sigma$ is c-planar and let $\Gamma(\sigma)$ be a c-planar drawing of $\sigma$. Denote with $v_{1}, \ldots, v_{n}$ the vertices of $\sigma$ starting from the first vertex of $\alpha$ and suppose, without loss of generality, that the length of $\alpha$ is greater or equal than the length of $\beta$. 


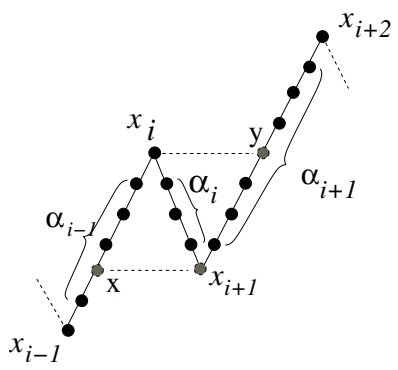

(a)

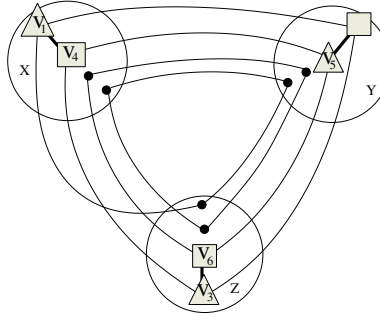

(b)

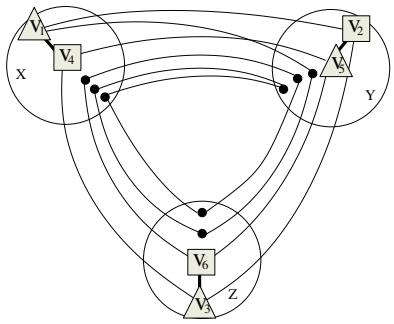

(c)

Fig. 3. Illustrations for the proofs of Lemma 2 (a), Lemma 3 (b) and of Lemma 4 (c).

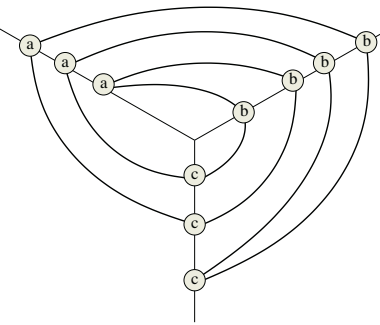

(a)

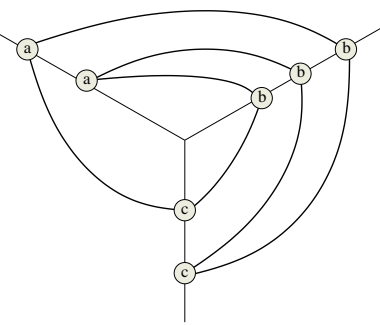

(b)

Fig. 4. The construction of a c-planar drawing for a cycle $\sigma$ when Balance $(\sigma)=0$ (a) and when $\operatorname{Balance}(\sigma)=3(\mathrm{~b})$.

Consider the relative position of $v_{1}$ and $v_{4}$ in $\Gamma$ in their cluster $X$ (see Fig. 3.c). We have that the two edges incident on $v_{4}$ separate $v_{1}$ from the rest of the vertices of $X$. Thus, it is possible to join $v_{1}$ and $v_{4}$ with an edge $\left(v_{1}, v_{4}\right)$ that is entirely contained into the cluster $X$ and that preserves the planarity of the drawing. Analogously, it is possible to join vertices $v_{2}$ and $v_{5}$ in cluster $Y$ with the edge $\left(v_{2}, v_{5}\right)$ and vertices $v_{3}$ and $v_{6}$ in cluster $Z$ with the edge $\left(v_{3}, v_{6}\right)$.

A contradiction arises since a subgraph that is a subdivision of $K_{3,3}$ can be found in the drawing. In fact, exploiting the edges of $\sigma$ and the edges introduced above, each vertex in $\left\{v_{1}, v_{3}, v_{5}\right\}$ is connected to all vertices in $\left\{v_{2}, v_{4}, v_{6}\right\}$. Vertex $v_{1}$ is directly connected to $v_{2}$ and to $v_{6}$ with edges of $\sigma$, while it is connected to $v_{4}$ with edge $\left(v_{1}, v_{4}\right)$; vertex $v_{3}$ is directly connected to $v_{2}$ and to $v_{4}$ with edges of $\sigma$, while it is connected to $v_{n}$ with edge $\left(v_{3}, v_{n}\right)$; finally, vertex $v_{5}$ is directly connected to $v_{4}$ with an edge of $\sigma$, it is connected to $v_{n}$ with a path in $\sigma$, and it is connected to $v_{2}$ with edge $\left(v_{2}, v_{5}\right)$.

Because of Lemma 2, Lemma 3, and Lemma 4, the problem of testing whether a 3 -cluster cycle $\sigma$ is c-planar can be reduced to the problem of computing Balance $(\sigma)$. Since it is easy to compute Balance $(\sigma)$ in linear time (see Section 2), the following theorem holds.

Theorem 1. Given an n-vertex 3-cluster cycle, there exists an algorithm to test if it is c-planar in $O(n)$ time.

In what follows we introduce a simple algorithm which guarantees the computation of a c-planar drawing of a 3-cluster cycle, if it admits one, in linear time. Consider a 
3-cluster cycle $\sigma$ with Balance $(\sigma) \in\{0,+3\}$. Set a counter to zero. Visit $\sigma$ starting from the first vertex and adding (subtracting) one unit to the counter when passing from $x$ to $y$, where $x \prec y(y \prec x)$. Without loss of generality we will assume that the counter never reaches a negative value. Otherwise, we can replace $\sigma$ with an equivalent cyclic permutation of it that has the above property and that can be obtained in linear time. Let $K$ be the maximum value assumed by the counter during the visit.

We say that a vertex of $\sigma$ belongs to the $k$-th level iff the counter has value $k$ when reaching such a vertex. The first vertex of $\sigma$ belongs to level 0 . Note that each level contains vertices of the same cluster. Also, vertices belonging to level $k$ and level $k+3$ belong to the same cluster. We denote with $\left.\sigma\right|_{k}$ the sequence $\sigma$ restricted to level $k$, obtained from $\sigma$ by deleting all the vertices not belonging to the $k$-th level.

We construct a saturator in the following way. For each level $k \in\{0, \ldots, K\}$, we connect with an edge each pair of consecutive vertices of $\left.\sigma\right|_{k}$. For each level $k \in$ $\{0, \ldots, K-3\}$, we insert an edge connecting the first vertex of $\left.\sigma\right|_{k}$ with the last vertex of $\left.\sigma\right|_{k+3}$.

Now we show that the graph composed by the cycle and the saturator is planar by providing a planar drawing of it (see Fig. 5). First, we arrange all the vertices of $\sigma$ on a grid: the x-coordinate of a vertex is its position in $\sigma$ and the y-coordinate is its level. Then, we draw each edge of the cycle (excluding the one connecting the first and the last vertex of $\sigma$ ) with a straight segment without introducing intersections. Second, for each level $k \in\{0, \ldots, K\}$, we draw those edges of the saturator that connect pairs of consecutive vertices of $\left.\sigma\right|_{k}$ with straight segments without introducing intersections. Note that, the sequence of the clusters at levels $0, \ldots, K-3$ is the same sequence as that of the clusters at levels $3, \ldots, K$. Also, at this point of the construction, for each $k \in\{0, \ldots, K\}$ the first and the last vertices of $\left.\sigma\right|_{k}$ are on the external face. Hence, the drawing can be completed without intersections by adding, for each level $k \in\{0, \ldots, K-3\}$, the edge of the saturator connecting the first vertex of $\left.\sigma\right|_{k}$ with

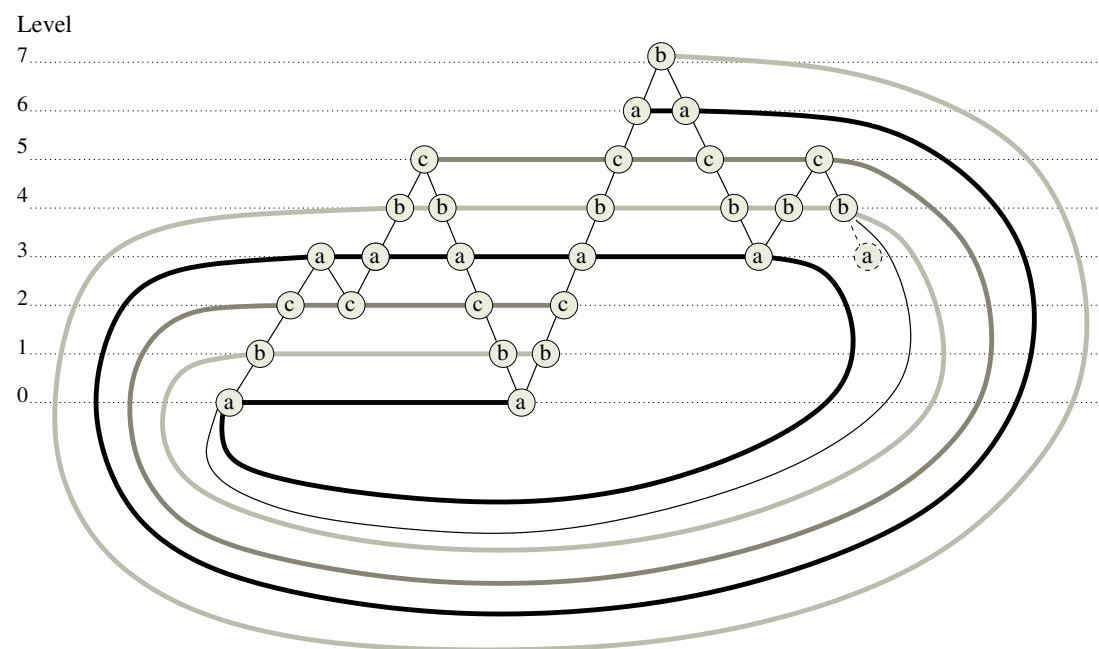

Fig. 5. The construction of a c-planar drawing of a 3-cluster cycle $\sigma$ in the case in which Balance $(\sigma)=3$. 
the last vertex of $\left.\sigma\right|_{k+3}$ as shown in the example of Fig. 5. Finally, since the first and the last vertex of $\sigma$ are on the same face, they can be connected with a curve contained into such a face without introducing intersections. To explicitly represents clusters as simple closed regions starting from the saturator we select a region of the plane at small distance (strictly greater than zero) from each saturator edge and delete the saturator.

It is easy to implement the above algorithm to work in linear time by building the lists of vertices for each level while visiting $\sigma$. Notice that $K$ is bounded by the number of the vertices of the cycle.

Hence, we can state the following result.

Theorem 2. Given an n-vertex c-planar 3-cluster cycle $\sigma$, there exists an algorithm that computes a c-planar drawing of $\sigma$ in $O(n)$ time.

From the above construction we also have the following.

Theorem 3. A c-planar 3-cluster cycle admits a saturator that is the collection of three disjoint paths.

If we consider the representation of 3-cluster cycles as strings, it is possible to show, in terms of formal grammars, that the set of 3-cluster cycles is a regular set, while the set of c-planar 3-cluster cycles is generated by a context-free grammar [4].

\section{Cycles in Cycles of Clusters}

In this section we present a generalization of the results of Section 3. First, we generalize the results on 3-cluster cycles to the case of clusters that form a cycle whose length is greater than three. Second, we tackle the general problem of testing the c-planarity of a cycle that is clustered into a cycle of clusters that is in turn clustered into another cycle of clusters, and so on. Fig. 6.a shows c-planar clustered graph whose underlying graph is a cycle for which two levels of clusters are defined. Fig. 6.b puts in evidence the inclusion relationship between clusters of a given level and clusters of the level directly above it. The same figure shows also that the clusters of each level form a cycle.

We start by introducing preliminary assumptions and definitions. We consider clustered graphs $C(G, T)$ in which all the leaves of the inclusion tree $T$ have the same distance from the root (we call depth that distance). A clustered graph which has not this property can be easily reduced to this case by inserting "dummy" nodes in $T$. Hence, from now on we consider only inclusion trees whose leaves are all at the same depth. We define as $G^{l}\left(V^{l}, E^{l}\right)$ the graph whose vertices are the nodes of $T$ at distance $l$ from its root, and an edge $(\mu, \nu)$ exists if and only if an edge of $G$ exists incident to both $\mu$ and $\nu$.

For example, $G^{0}$ has only one vertex and $G^{L}$, where $L$ is the depth of the tree, is the underlying graph $G$ of $C(G, T)$. We label each vertex $\nu$ of $G^{l}$ with the cluster (corresponding to a vertex of $G^{l-1}$ ) which $\nu$ belongs to. If $G^{l}$ is a cycle, then it is possible to identify $G^{l}$ with the cyclic sequence of the labels of its vertices. If also $G^{l-1}$ is a cycle, we consider the labels of $G^{l}$ cyclically ordered according to the order they appear in $G^{l-1}$. At this point, Balance $\left(G^{l}\right)$ can be defined as in Section 3 and can assume values $0, k, 2 k, 3 k, \ldots$ where $k$ is the length of $G^{l-1}$. 


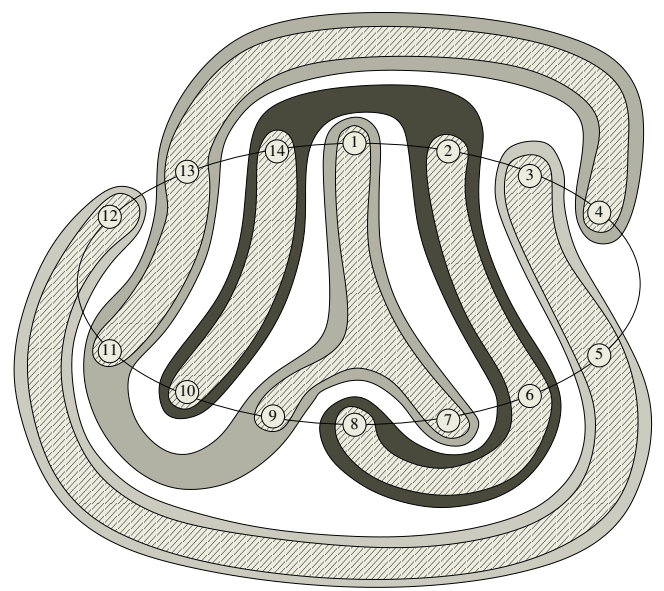

(a)

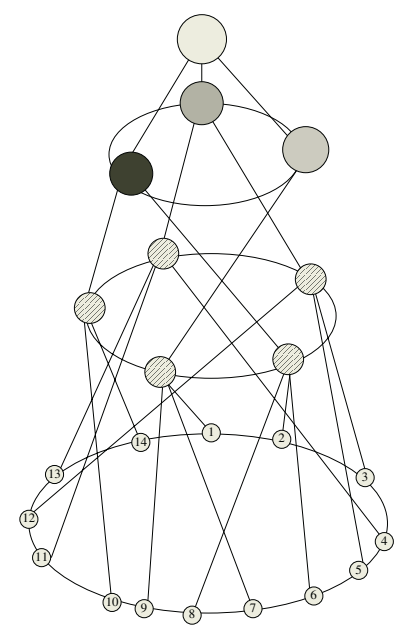

(b)

Fig. 6. A clustered graph where at each level of the inclusion tree the nodes form a cycle. (a) A cplanar drawing. (b) The inclusion tree augmented with edges that put in evidence the adjacencies between nodes at the same level.

According to the above definitions a 3-cluster cycle is a clustered graph where $T$ as depth $2, G^{2}$ is a cycle and $G^{1}$ is a cycle of length 3 . In fact, the results of Section 3 can be extended to the case in which $G^{1}$ is a cycle of an arbitrary length.

Theorem 4. Given an n-vertex clustered graph $C(G, T)$, such that $T$ has depth 2 and $G^{1}$ and $G^{2}$ are cycles, there exists an algorithm to test if $C$ is c-planar in $O(n)$ time. If $C$ is c-planar, a c-planar drawing of $C$ can be computed in $O(n)$ time.

Proof Sketch: The proof exploits the same considerations and constructions of Theorems 1 and 2. If the length of $G^{1}$ is $k$ then $C$ is c-planar iff Balance $\left(G^{2}\right) \in\{0, k\}$. In order to find a c-planar drawing of $C$, if it exists, the same strategy described in Section 3 can be applied, where, since in the construction depicted in Fig. 5 vertices belonging to level $j$ and level $j+k$ belong to the same cluster, an edge of the saturator is added between the first vertex of level $j$ and the last vertex of level $j+k$ instead of between the first vertex of level $j$ and the last vertex of level $j+3$.

Let $C(G, T)$ be a clustered graph and $l$ be an integer between 1 and $L$, where $L$ is the depth of $T$. Clustered graph $C^{l}\left(G, T^{l}\right)$ is obtained from $C$ by replacing $T$ with a tree $T^{l}$ obtained from $T$ by connecting all the nodes at depth $l$ with the root and deleting all the nodes having depth greater than zero and less than $l$. The c-planarity of $C^{l}$ can be used to study the c-planarity of $C^{l-1}$, as is shown in the following lemma.

Lemma 5. Let $C(G, T)$ be a clustered graph and $l$ be an integer between 1 and $L$, where $L$ is the depth of $T$. Let $C^{l}$ be c-planar, $G^{l}$ be a cycle, and $G^{l-1}$ be a cycle of length k. $C^{l-1}$ is c-planar iff Balance $\left(G^{l}\right) \in\{0, k\}$.

Proof Sketch: First, we prove that if Balance $\left(G^{l}\right) \in\{0, k\}$, then $C^{l-1}$ is c-planar. Since Balance $\left(G^{l}\right) \in\{0, k\}$, then it exists a planar drawing of $G^{l}$ augmented with 
the edges of a saturator connecting vertices of $G^{l}$ with the same label. Those edges can be added to the internal or external face of cycle $G^{l}$ according to the output of the algorithm described in Section 3. Let $\Gamma_{C^{l}}$ be a c-planar drawing of $C^{l}$. Since $G^{l}$ is a cycle, there exist in $\Gamma_{C^{l}}$ exactly two faces containing vertices belonging to all the clusters corresponding to vertices of $G^{l-1}$. Call such faces internal and external face arbitrarily. A c-planar drawing $\Gamma_{C^{l-1}}$ can be constructed by adding to $\Gamma_{C^{l}}$ an edge for each edge of the saturator of $G^{l}$ in such a way to place on the internal (external) face of $\Gamma_{C^{l}}$ the edges of the saturator that are added to the internal (external) face of $G^{l}$.

The second part of the proof shows that if $\operatorname{Balance}\left(G^{l}\right)$ is not in $\{0, k\}$ then then $C^{l-1}$ is not c-planar. Assume for a contradiction that $\operatorname{Balance}\left(G^{l}\right)$ is not in $\{0, k\}$ and a c-planar drawing of $\Gamma_{C^{l-1}}$ exists.

By using similar arguments as in the proofs of Lemmas 3 and 4, a subdivision of a $K_{3,3}$ can be found where the vertices of the subdivision are actually vertices of $G^{l}$, that is, clusters of $C$.

Lemma 6. Let $C=(G, T)$ be a clustered graph and let be $l$ an integer between 1 and $L$, where $L$ is the depth of $T$. If $C^{l}$ is not c-planar, then $C^{0}=C$ is not c-planar.

Proof Sketch: If $C^{l}$ is not c-planar, there is a subdivision of $K_{3,3}$ or $K_{5}$ in the graph G augmented with the edges of the saturator of $C^{l}$. The same obstruction can be found in the graph $G$ augmented with the edges of saturator of $C^{0}$; hence $C^{0}$ can not be c-planar.

Theorem 5. Given an n-vertex clustered graph $C(G, T)$, such that $T$ has depth $L$ and, for $l>0, G^{l}$ is a cycle, there exists an algorithm to test if $C$ is c-planar in $O(L n)$ time.

Proof Sketch: The proof is based on iteratively applying, level by level, Lemma 5 to the clustered graphs $C^{l}$ for $l=L, L-1, \ldots, 2$. Since each test can be performed in $O(n)$ time, the statement follows.

Theorem 6. Given an n-vertex clustered graph $C(G, T)$, such that $T$ has depth $L$ and, for $l>0, G^{l}$ is a cycle, if $C$ is c-planar there exists an algorithm to compute a c-planar drawing of $C$ in $O(L n)$ time.

Proof Sketch: The proof of Lemma 5 is a constructive one. Thus, by applying, level by level, Lemma 5 starting from level $L$ to level 1, a c-planar drawing of $C$ can be obtained. Since each step may be performed in $O(n)$ the statement follows.

\section{Conclusions and Open Problems}

In this paper we studied a peculiar family of non-connected clustered graphs. Namely, we studied clustered graphs whose underlying graph is a simple cycle. Besides the general problem of stating the complexity of the c-planarity testing of non-connected clustered graphs, several other problems remain open.

Are there other families of non-connected clustered graphs whose c-planarity can be efficiently assessed and whose underlying graph has a simple structure? For example, 
what happens if the underlying graph is a tree? It is easy to show that a two-level clustered graph whose underlying graph $G^{2}$ is a path and such that graph $G^{1}$ is a cycle, is c-planar. It is also easy to find an example of a two level clustered graph whose underlying graph $G^{2}$ is a tree, such that $G^{1}$ is a cycle and that is not c-planar.

Suppose that the underlying graph has a fixed embedding. Can this hypothesis simplify the c-planarity testing?

Can the techniques introduced in this paper be combined with techniques known in the literature for devising tools able to handle the c-planarity testing and embedding problem for more complex families of clustered graphs?

\section{References}

1. T. Biedl, M. Kaufmann, and P. Mutzel. Drawing planar partitions II: HH-Drawings. In Workshop on Graph-Theoretic Concepts in Computer Science (WG'98), volume 1517, pages 124-136. Springer-Verlag, 1998.

2. T. C. Biedl. Drawing planar partitions III: Two constrained embedding problems. Tech. Report RRR 13-98, RUTCOR Rutgen University, 1998.

3. S. Cornelsen and D. Wagner. Completely connected clustered graphs. In Proc. 29th Intl. Workshop on Graph-Theoretic Concepts in Computer Science (WG 2003), volume 2880 of LNCS, pages 168-179. Springer-Verlag, 2003.

4. P. F. Cortese, G. Di Battista, M. Patrignani, and M. Pizzonia. Clustering cycles into cycles of clusters. Technical Report RT-DIA-91-2004, Dipartimento di Informatica e Automazione, Università di Roma Tre, Rome, Italy, 2004.

5. G. Di Battista, W. Didimo, and A. Marcandalli. Planarization of clustered graphs. In Proc. Graph Drawing 2001 (GD'01), LNCS, pages 60-74. Springer-Verlag, 2001.

6. G. Di Battista, P. Eades, R. Tamassia, and I. G. Tollis. Graph Drawing. Prentice Hall, Upper Saddle River, NJ, 1999.

7. S. Even. Graph Algorithms. Computer Science Press, Potomac, Maryland, 1979.

8. Q. W. Feng, R. F. Cohen, and P. Eades. How to draw a planar clustered graph. In Ding-Zhu Du and Ming Li, editors, Proc. COCOON'95, volume 959 of LNCS, pages 21-30. SpringerVerlag, 1995.

9. Q. W. Feng, R. F. Cohen, and P. Eades. Planarity for clustered graphs. In P. Spirakis, editor, Symposium on Algorithms (Proc. ESA '95), volume 979 of LNCS, pages 213-226. SpringerVerlag, 1995.

10. C. Gutwenger, M. Jünger, S. Leipert, P. Mutzel, M. Percan, and R. Weiskircher. Advances in $C$-planarity testing of clustered graphs. In Stephen G. Kobourov and Michael T. Goodrich, editors, Proc. Graph Drawing 2002 (GD'02), volume 2528 of LNCS, pages 220235. Springer-Verlag, 2002.

11. J. E. Hopcroft and J. D. Ullman. Introduction to Automata Theory, Languages, and Computation. Addison-Wesley, 1979. 